new approach - which was deployed in 2015 - will be available early 2016.

\section{FIREARM LEGISLATION CAN REDUCE FIREARM-RELATED INJURIES IN CHILDREN}

$\underline{A B}$ Van As, NM Campbell, JG Colville, Y van der Heyde, A Numanoglu. Red Cross War Memorial Children's Hospital, University of Cape Town

\subsection{6/injuryprev-2016-042156.467}

Background Violence and firearms are common features of South African society: the leading cause of death being those resulting from violence and homicide, with firearms being ranked as a leading external cause of non-natural deaths. The Red Cross Children Hospital is the only hospital in Africa with a dedicated trauma unit for children and has been dealing with gunshot wounds since 1991.

Methods A retrospective review of firearms injuries which presented to the Red Cross Children's Hospital between 1991 and 2011 was performed. Data recorded included the folder numbers; sex; date of birth; age; date of presentation; date discharged and inpatient stay; firearm type; number of shots; circumstances; injury sites; injury type, treatment; resulting morbidities and survival.

Results 441 children presented with firearm injuries during the review period. The results showed a steady decrease in incidence from 2001-2011. There was a greater incidence amongst older children and males. Contrary to studies in adults, the majority of children were shot unintentionally, as innocent bystanders and in crossfire. During the first decade (1991-2000) there was a gradual increase in incidence of children suffering from firearms injuries with time, peaking in the year 2000 , while the number of gunshots gradually declined during the second decade (20012010). The study showed a decline in total firearm injuries in children since 2001, coinciding with the legislation introduced in 2004 and the changes in government opinion since 2000. Mortality also reduced significantly from the previous study (6\% to 2.6\%), as did the total number of in-patient days (1063 to 635). Conclusions This study showed a significant reduction in the number of children presenting with a firearm-related injury after the implementation of the New Firearm Bill. Mortality rate and in-patient stay were also significantly reduced. This study shows the impact that the Firearms Control Act has had in terms of paediatric firearm-related injury and provides clear evidence of the important role civil society mobilisation can play in the prevention of fire-arm related injuries in children.

\section{Child Maltreatment}

\section{Post Mon 1.8}

\section{PREVALENCE OF CHILD ABUSE AMONG CHILDREN AGED 11 TO 17 YEARS OLD IN COMMUNITY SETTINGS OF KARACHI, PAKISTAN}

\footnotetext{
${ }^{1}$ Maryam Lakhdir, ${ }^{1}$ Masood Kadir, ${ }^{1}$ Iqbal Azam, ${ }^{2}$ Yasmin Parpio, ${ }^{3}$ Uzma Khan, ${ }^{4}$ Junaid Razzak. 'Department of Community Health Sciences, Aga Khan University, Pakistan; ${ }^{2}$ Department of School of Nursing and Midwifery, Aga Khan University, Pakistan; ${ }^{3}$ Department of Emergency Medicine, Aga Khan University, Pakistan; ${ }^{4}$ The Johns Hopkins Medical Institution, Maryland, USA
}

10.1136/injuryprev-2016-042156.468
Background Harsh disciplinary practices are considered common in developing countries. However, scientific evidence lacks to support this. Primary aim of this study was to determine the prevalence of different forms (physical, emotional maltreatment and neglect) of child abuse in Karachi, Pakistan.

Methods A cross sectional survey of 800 dyads (children ranging from 11-17 years old and their parent) were selected from 32 clusters of Karachi, using multistage cluster sampling between December 2014 to March 2015. A structured questionnaire adopted from International society of child abuse and neglect (International child abuse screening tool for parent and child). Prevalence of child maltreatment was estimated by mean child abuse score.

Results Mean score were $51.7+11.9$ (child), $77.9+20.2$ (mother) and $63.6+17.3$ (father). Both parent and child responses revealed similar prevalence $(43 \%)$ of child maltreatment. The mother's responses showed a prevalence of physical (42\%), emotional (50\%) maltreatment and neglect $(5.4 \%)$ whereas, father's responses showed a prevalence of physical (39\%), emotional (46\%) maltreatment and neglect (9.7\%). The child's experiences showed a prevalence of physical (49\%), emotional (50\%) maltreatment and neglect (57\%). A substantial proportion of participants were exposed to at-least one form of maltreatment on mother (58\%), father (54\%) and child (74\%) responses, of which all three maltreatment forms experienced by children were $4 \%, 9 \%$ and $31 \%$ on mother, father and child responses respectively.

Conclusions This study reflects hidden extent of child maltreatment and poly-victimisation in Karachi. Our findings provide evidence to raise public awareness about malpractices and highlight the need to develop positive parenting program.

\section{PREVALENCE OF VIOLENCE AGAINST CHILDREN IN CHINA}

${ }^{1}$ Gao Xin, ${ }^{2} Z$ hu Xu, ${ }^{1}$ Wang Linhong, ${ }^{1} J i n$ Ye, ${ }^{1}$ Duan Leilei, ${ }^{2}$ Robert Scherpbier. 'National Centre for Non-Communicable Disease Control and Prevention, China Centre for Disease Prevention and Control,Beijing, 100050,China; ${ }^{2}$ United Nations Children's Fund UNICEF Office for China, Beijing, 100600, China

\subsection{6/injuryprev-2016-042156.469}

Background Violence against children (VAC) prevention is the priority of public health; and to master the prevalence state and risk factors of VAC is the base to develop the prevention strategies. This study aimed to obtain the prevalence features and to find the risk factors of VAC in China, and provide basis and formulate the prevention strategies.

Methods The death data of VAC was from The National disease surveillance points system (DSP) during 2006-2012. There were 161 surveillance points in DSP distributed in all the 31 provinces in China. The total population in this system was more than 73 million. We analysed the data from DSP about children aged 017 died from violence (ICD-10: V01-Y89). The VAC hospital information was from The National Injury Surveillance System (NISS) during 2006-2013. This system included 126 hospitals from 43 surveillance points. We analysed the data from NISS about children aged $0-17$ caused by VAC with the parameter of "intentional" injury.

Results The trend of death caused by VAC had declined overall; it was reduced to $0.52 / 100,000$ in 2012 from $0.85 / 100,000$ in 2006. The male VAC mortality was higher than female, and the rural was higher than urban. Blunt injury was the leading maltreatment injury (63.35\%), followed by sharp injury (11.09\%). 
The main violence occurrence place against infant was home. Home and public residential institution were the main VAC occurrence places for 1-4 years old children, and school was the main place of violence against the children over 5 years old.

Conclusions The declining death rate of VAC may be related to the social benign development. The related child protection laws and regulations should be implemented further and the prevention programs targeting the domestic violence and the school violence should be prioritised.

\section{ADVERSE CHILDHOOD EXPERIENCES STUDY AMONG UNIVERSITY STUDENTS IN TURKEY}

${ }^{1}$ Betul Ulukol, ${ }^{2}$ Akfer Kahilogullarl, ${ }^{3}$ Dinesh Sethi, ${ }^{4}$ Sevtap Velipasaoglu Guney, ${ }^{1}$ Ozdecan Bezirci Odek, ${ }^{5}$ Suvat Parin, ${ }^{6}$ Murat Yopbas, ${ }^{7}$ Sadik Aksit, ${ }^{7}$ Feyza Umay Koc, ${ }^{1}$ Kenan Kose, ${ }^{6}$ Gamze Can. ${ }^{1}$ Ankara University, Turkey; ${ }^{2}$ DIskapı Yildirim Beyazit Resarch Hospital, Turkey; ${ }^{3}$ World Health Organisation; ${ }^{4}$ Akdeniz University, Turkey; ${ }^{5}$ Yuzuncu Yil University, Turkey; ${ }^{6}$ Karadeniz Technical University, Turkey; ${ }^{7}$ Ege University, Turkey

\subsection{6/injuryprev-2016-042156.470}

Background There is a salient relationship between exposure to childhood traumatic events and negative health behaviours, and health status. The aim of this study was to identify the prevalence of adverse childhood experiences (ACEs) in a group of university students in Turkey and to evaluate the association of ACEs, with some health consequences including health risk behaviours.

Methods This is a descriptive cross-sectional study. 2257 students of 5 universities in Turkey were enrolled into the study between the years 2012-2013. A questionnaire modified from the ACE Questionnaire developed by CDC and Kaiser Permanente was used in the study. The questionnaire includes 53 questions on sociodemographic characteristics, household dysfunction, childhood maltreatment, health risk behaviours, somatic complaints and health status.

Results $47.9 \%$ of the 2257 respondents were male and $52.1 \%$ were female. The mean age of respondents was 20.1 years. The overall prevalence of childhood physical abuse, sexual abuse, emotional abuse, emotional neglect and physical neglect was $21.1 \%$, $7.9 \%, 9.8 \%, 8.8 \%$ and $5.7 \%$ respectively. $5.2 \%$ of respondents had divorced or separated parents. The overall prevalence of depression or suicide attempt, problem alcohol use, history of street drug use and involvement in crime or imprisonment in household members was $9.3 \%, 6.4 \%, 3.4 \%$ and $10.3 \%$ respectively. The ACE scores indicate that half of all respondents had a history of at least one ACE. ACE score was positively associated with health risk behaviours. The risk of smoking, harmful alcohol using and drug using increases dependently on the ACE score. Some health problems particularly emotional problems were associated with ACE score of the participants.

Conclusions This study suggests that ACE prevalence, health risk behaviours and certain health problems are high in a group of young adults in Turkey. The data from this study does not only provide information about the magnitude of the problem but also evidence that underlines the need to prioritise child maltreatment besides.

\section{THE FAMILY HEALTH, FUNCTIONING AND CHILD MALTREATMENT RISK OF FAMILIES EXPECTING A BABY}

\footnotetext{
1,2Eija Paavilainen, ${ }^{3}$ Sari Lepistö, ${ }^{1,3}$ Mika Helminen, ${ }^{1}$ Noora Ellonen. ${ }^{1}$ University of Tampere, Finland; ${ }^{2}$ Etelä-Pohjanmaa Hospital District, Finland; ${ }^{3}$ Pirkanmaa Hospital District, Finland
}

Background The purpose of this study is to describe the family health, functioning, social support and the child maltreatment risk and associations between them in families expecting a baby. It is crucial for welfare of children, to find families needing special support before the pattern of maltreatment has been developed within the family.

Methods The child maltreatment risk in families was measured using the Child Abuse Potential Scale (CAP). Health, functioning and social support were measured using the Family Health, Functioning and Social Support Scale (FAFHES). Data were collected from mothers and spouses at 30 weeks of pregnancy. Data were analysed by multivariate logistic modelling for explaining the child maltreatment risk level of families and for examining the associations between health, functioning, social support and the risk level.

Results Data included 380 families, 136 of them were expecting their first baby and 78 families had an increased risk for child maltreatment. Heightened risk was associated with the age of the spouse, the mother's education, the mental health problems of the spouse's father, the mother's concern about her spouse's drinking, and the mother's difficulties in talking about the family's problems. Child maltreatment risk was associated with family functioning and health. Families with risk received a little less support from maternity clinics.

Conclusions Families with child maltreatment risk and related factors were found. This knowledge can be applied for supporting families both during pregnancy and after the baby is born. The research continues as a part of Family Violence Research Project, by assessing the possible changes in family situation, while the baby is about 12 months of age.

\section{EFFECT OF SIBLINGS IN A BELGIAN SURVEILLANCE SYSTEM OF CHILD MALTREATMENT: COMPARISONS OF SEVERAL STATISTICAL METHODS}

${ }^{1}$ Christelle Senterre, ${ }^{1}$ Alain Levêque, ${ }^{2}$ Brigitte Vanthournout, 'Michèle Dramaix. ${ }^{1}$ Université Libre De Bruxelles, School of Public Health, Research Centre in Epidemiology, Biostatistics and Clinical Research, Belgium; '2SOS Enfants ULB, CHU Saint Pierre, Belgium

\subsection{6/injuryprev-2016-042156.472}

Background Over time, the circumstances encountered in case of child maltreatment, can be quite complex and then, can lead to methodological questions for the analysis of the data. Based on data coming from 395 children hospitalised, alone $(66.1 \%)$ or in siblings (33.9\%), in a paediatric ward between 2007 and 2012 for mistreatment or because of a severe risk of mistreatment, the aims of this study were to quantify the degree of similarity between sibling members, to study the differences between children hospitalised alone or with siblings and to compare four statistical methods for the analyses of the associated factors of mistreatment.

Methods To quantify the degree of similarity between sibship members, the Snijders and Bosker intracluster correlation coefficient was calculated and the statistical methods used were the logistic regression and GEE, both without and with robust standard error.

Results Almost all intracluster correlation coefficients were large, meaning that the sibling's members have a higher degree of similarity between them. The odds ratios were not exactly the same between the two models and the robust standard errors where almost always higher than the model-based standard errors in both logistic and GEE models leading to wider confidence intervals. 